\title{
AI Enhanced Alarm Presentation for Quality Monitoring
}

\author{
Satu Tamminen*, Xiaoli Liu*, Henna Tiensuu*, Eija Ferreira*, Esa Puukko ${ }^{\dagger}$ and Juha Röning* \\ *Biomimetics and Intelligent Systems Group \\ University of Oulu \\ Oulu, Finland \\ Email: satu.tamminen@oulu.fi \\ ${ }^{\dagger}$ Outokumpu Stainless $O y$ \\ Tornio, Finland
}

\begin{abstract}
This paper presents an AI based method for improving poorly performing quality prediction models. The method improves automatically the usability of the low quality alarm predictions in the web based quality monitoring tool that provides decision support for users. The tool enables the utilization of the models that suffer from the lack of information because of a long time gap to the predicted future. The reliability of the presented alarms in a monitoring tool will be improved by reducing the amount of false alarms.
\end{abstract}

\section{Introduction}

Smart city focuses on applying next-generation technology to every-day life, environmental protection, public security, city services and other fields. The concept is based on the use of information and communication technology to sense, analyse and integrate the key information to the core systems in running cities. The technology of intelligent analysis and decision support is one of the key challenges when constructing a smart city [1]. Solutions to this challenge can be searched from the industrial applications where the intelligent data analysis and decision support already exist in process-wide or even plant-wide use.

Smart cities consist of smart sensing components that can be located in infrastructures, buildings, electricity and water distribution, transportation, services, surveillance and healthcare [2]. By making a city smart it is possible to mitigate the problems generated by the urban population growth and rapid urbanization. Smart city is more efficient, sustainable, equitable and livable [3].

Advanced systems to improve and automate processes within a city may help to save the scarce resources. We need more intelligent systems for water and electricity distribution, buildings and homes, energy sensing, demand prediction based on weather conditions, occupancy and user context, indoor monitoring, smart building evacuation, monitoring bridges and seismic activity, environmental monitoring, intelligent transportation, public services, such as, law enforcement and fire fighting, and health care [2]. Public spaces monitoring enables the providing of services to the citizens. Alert announcers are the application layer that notify the users of specific events, warnings and alarms [4].
As the increasing number of sensors enabling IoT produce information that can be utilized for providing services to the citizens, it is still not common to have advanced platforms of systems for live monitoring and inferring of urban process parameters. With advanced sensing and computation capabilities, it is possible to gather and evaluate the data in real time to extract the information and convert it to usable knowledge, which will turn the city smart [5].

Sensors are a crucial component of any intelligent control system [2]. Modern manufacturing process produces constantly collected sensor data in massive amounts. Today's industry builds its competitiveness on quality management, delivery reliability and resource efficiency, which are dependent on the effective usage of the data collected from different sources.

Knowledge engineering and data mining have enabled the development of new types of manufacturing systems. Future manufacturing is able to adapt to demands of agile manufacturing, including rapid response to changing customer requirements, concurrent design and engineering, lower cost of small volume production, outsourcing of supply, distributed manufacturing, just-in-time delivery, realtime planning and scheduling, increased demands for precision and quality, reduced tolerance for errors, in-process measurements and feedback control [6]. Smart manufacturing will bring solutions to existing challenges, but the current industry utilizes generally the information from its environment and in best cases only the first level of knowledge (Figure 1). The progress in industrial data utilization is enabled with novel intelligent data processing methods.

There are various widely used methods that are able to monitor the current process variables or product properties factor-wise. Control charts, statistical process control (SPC) and automatic process control (APC) are in everyday use for process monitoring and adjustment. Statistical models give another angle to quality monitoring, as they can be used to predict the future outcome of a process, which in its turn enables planning of the process and the production as a whole [7], [8]. Statistical prediction models have been utilized also in smart city related applications; a driving coach system can assist the driver for more fuel- efficient driving by predicting the fuel consumption with city map 


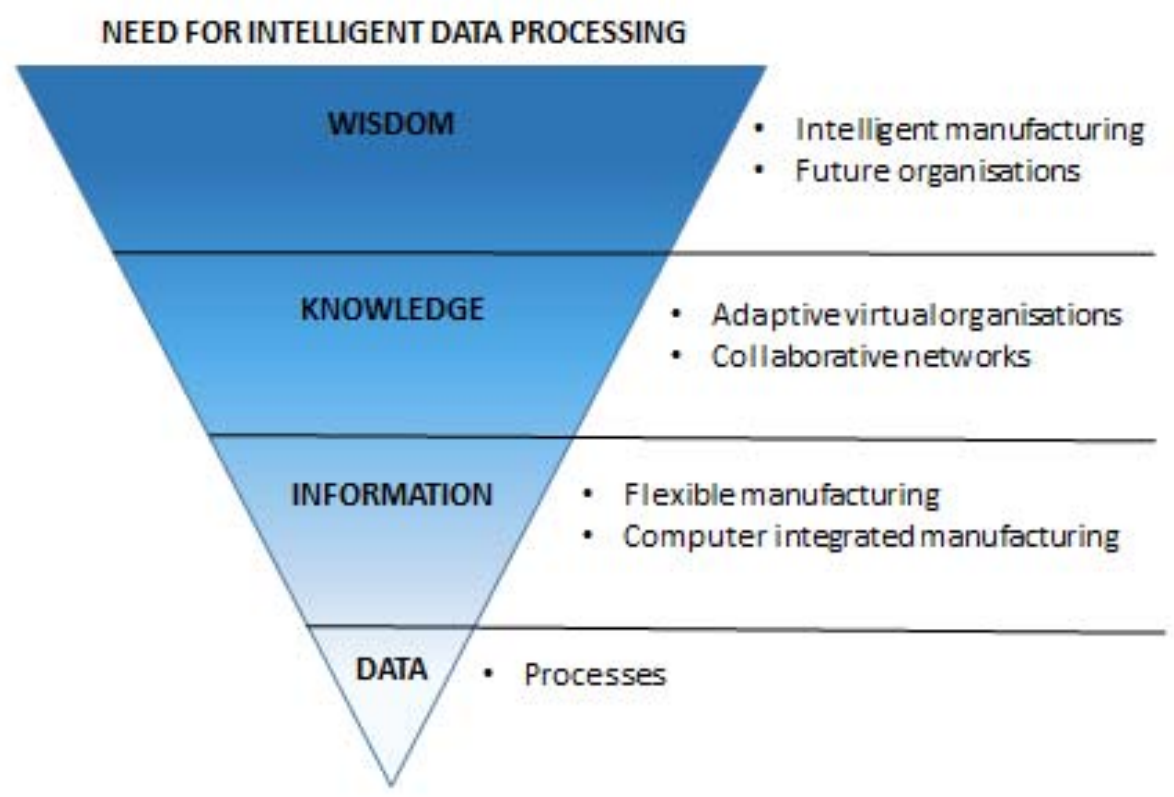

Figure 1. The evolution of data to knowledge requires novel methods for intelligent data processing that enable the shift to smart manufacturing.

data, weather and context information [9].

However, the full potential of the essential knowledge within the data cannot be utilised with limited capacity to process the incessant information flow. In some cases, the accuracy of the model may not be satisfactory due to the absence of related data. With the help of AI, it might be possible to benefit even from an ill-performing model by comparing prediction results to existing knowledge about the data.

Manufacturing has benefited from the field of data mining in several areas, including engineering design, manufacturing systems, decision support systems, shop floor control and layout, fault detection, quality improvement, maintenance, and customer relationship management [10]. Information collected from large number of different sources is difficult to comprehend, and thus in today's manufacturing, there is a need for intelligent data processing. Decision support systems (DSS) become intelligent when combined with AI tools such as fuzzy logic, case-based reasoning, evolutionary computing, artificial neural networks (ANN), and intelligent agents [11], [12].

Kano and Nakagawa suggest that when product quality improvement is pursued, the process monitoring system should have at least following functions: it should be able to predict product quality from operating conditions, to derive better operating conditions that can improve the product quality, and to detect faults or malfunctions for preventing undesirable operation [13]. Usability of the quality models correlates with the benefits, the tool can afford. Xu et al. show that when the massive amount of real-time data flow is to be analysed, currently strong big data analytics skills are needed from the end user [14]. Yet, the employment of experts concentrate on the core area of the industry, which in turn, generate a demand for intelligent tools for decision support.

Trust and dependence on automatic decision support systems may have negative consequences, such as, over reliance and misuse of the system. In the case of very low level of trust, the system may be disused entirely [15], [16], [17]. Thus, the reliability of the system has a key role in the system design.

In this paper, we present a method for AI enhanced models in quality monitoring. Our intelligent support for decision-making combines the predictions of the quality models with the diagnostics of the process data. Especially during online monitoring, when the reaction time is limited, it is important to deliver the automatic interpretation of the results to the user. The method helps to reduce the amount of false positives in prediction, and thus, to increase the reliability of the tool and to reduce the unnecessary cognitive load of the user.

\section{AI enhanced quality model}

\subsection{Prediction model}

In industrial applications, high nonlinearity and numerous interactions between process settings challenge the model performance. Furthermore, for the user the value is not just in the prediction but also in the information about the effects of the process variables to the predicted quality property. Additionally, with online system the functionality of the tool will suffer, if the models were not capable of processing observations with missing data that cannot be fully avoided. 
Over the years, neural networks have been a popular method for modelling data with complex relations between variables [18], [19]. Lately, ensemble algorithms have risen to challenge them with equal accuracy, faster learning, tendency to reduce bias and variance, and also lower tendency to over-fit. Seni and Elder state that ensemble methods have been called the most influential development in Data Mining and Machine Learning in the past decade [20].

Gradient boosting machines are a family of powerful machine learning techniques that has been successfully applied to a wide range of practical applications [21]. Boosted regression trees are suitable for classification as well, and they are capable of handling different types of predictors and accommodating missing data, there is no need for prior transformation of variables, they can fit complex nonlinear relationships, and automatically handle interactions between predictors [22]. For quality prediction, we used the generalized boosted regression models (GBM). The details about the method can be found in [23].

\subsection{Model improvement}

Powerful modelling methods do not guarantee the reliable results, if the training data fails to represent adequately well the relationships of the observed phenomenon. However, the cost effects of the predictions can motivate to use the model anyway. With classification models, the tradeoff between sensitivity and specificity affects the model selection. In industrial applications, the presence of false positives will have cost effects as well as the failing in recognition of true positives, and thus, their number should be reduced.

For each product group, we collected a pool of good products. These products have a combination of highly influential process variables with safer value settings. We used actual products instead of aggregate information (e.g., average) of the variables. This way, the combination of variable settings is realistic. In this application, we divided the products to five groups based on the similarity of their production practices and mechanical properties. We selected the most influential process variables that will be used in similarity calculation with the help of GBM model.

We used following steps for reducing the number of false positives:

Step 1. A GBM model for selected quality property will be trained. The model will be utilised for quality prediction, and it will also provide information about the most influential process variables that affect the quality. When a prediction indicates a defect for a product, the process settings will be examined and the reliability of the prediction will be improved during the following steps.

Step 2. The most influential variables of the good products will be compared to the current product with predicted defect. For each variable, the distance to the safe zone will be calculated. The user could inspect the differences visually as well, but when reducing the number of false positives, the procedure of finding the variables not locating in the safe zone is automatic.
Step 3. The alarm for defect risk will be presented only, if the inspected product actually has alarming values for variables that have a high impact on the quality property. The user will get also information about the factors causing the risk, and thus, safer process settings can be selected according to the automatic recommendations.

\section{Experiments}

\subsection{Data}

In manufacturing, the cost effectiveness is one of the factors that affect the competitiveness. Our Methods proposed in this paper enable the reduction of costs that may be caused by the unnecessary shipping of the products between the plant sites and by the defects that may lead to rejection or quality grade reduction, for example.

The data was collected at the Outokumpu Stainless Oy in Tornio, Finland during 1.2.2016-4.4.2017 and it consists of 8026 observations with 98 variables, including process settings, chemical composition and information of the surface quality. Additional test set with 2441 observations was collected during 1.6-31.10.2017. The roughness located at the edge-zones of the stainless steel strip is a quality property that can be detected only after the polishing of the finished product. The defect can originate already during the hot rolling, but after that the product will go through cold rolling, different surface treatments and finally polishing, and these following process steps may also worsen the defect. The plants for hot and cold rolling locate in different countries, and thus, it would be crucial to find the products that do not meet the quality requirements already before the shipping.

There are several factors that reduce the accuracy of the prediction models. First of all, the prediction need to be done at the early state of the process steps, and thus some of the affecting variables have to be left out from the model. Also, some of the process variables cannot be measured accurately with available devices. Finally, the predicted quality variable is measured with inexact method. In this application, the roughness is detected with visual inspection made by humans, and the severity of the defect is evaluated with a nine-class rating scale. Only the information about the presence of the roughness defect was usable for prediction.

\subsection{Quality presentation}

During steel manufacturing, the process is monitored with a web-based online quality monitoring tool (QMT) that will give an alarm for products with increased risk for defected surface based on the quality prediction model [24]. Figure 2 presents the schematic figure of the QMT prototype. The transfer of the information from the manufacturing process to the end users is presented in the following four steps that are 1) data acquisition, 2) data storage, 3) information analysis and 4) information delivery. In most advanced visualizations in our tool, the information 
has been refined to knowledge with automatic interpretation of the results. We built the GBM models and intelligent visualizations of the rootcauses with free statistical program R.

For quality prediction model, we used $70 \%$ of the data for training and $30 \%$ for model validation. We secured the independence between training and validation data sets by selecting observations in batches. Also, the proportion of the product types was preserved. An independent test set of 2441 observations was used for final testing of the model.

The relative influence of each input variable in GBM models tells about the strength of their impact on the quality property. The ten most influential variables in roughness models can be found in Table 1 . We used this information when selecting the variables for further analysis of the results. GBM models provide also visualized impact of each input on the dependent variable with partial dependence plots (PDP). Figure 3 presents the effect of the most influential variable on the roughness. An increase on the specific force on the fifth pass will increase the surface roughness as well. PDP plots have educational value, when the user wants to learn more about the manufacturing process.

TABLE 1. RELATIVE INFLUENCE OF THE PROCESS VARIABLES IN THE ROUGHNESS MODEL.

\begin{tabular}{ll}
\hline Variable & Rel. influence \\
\hline Specific force 5 & 11.4665 \\
$\mathrm{Cr}$ & 11.0681 \\
Cropshear temp. & 8.4040 \\
$\mathrm{Nb}$ & 6.1541 \\
Shift of working roll 9 & 6.0464 \\
Bending 10 & 5.7175 \\
Ti & 5.1868 \\
Specific force 9 & 5.1124 \\
Specific force 3 & 5.0455 \\
Thickness 11 & 4.7733 \\
\hline
\end{tabular}

The results of the original prediction model are presented in Table 2, where defected products have value 1 and high quality products have value 0 . It can be seen that the model performance suffers from the factors that reduce the accuracy, the model either fails to recognise correctly a high number of defected products, or produces a high number of false alarms. The usability of the tool is poor, because, the high number of false alarms; in worst product groups the number of false positives exceeds the number of true positives.

TABLE 2. THE ORIGINAL PREDICTION MODEL PERFORMANCE IN TRAINING, VALIDATION AND TEST SETS.

\begin{tabular}{lll}
\hline & \multicolumn{2}{c}{ Measured } \\
& 0 & 1 \\
\hline Pred. 0 & 5007 & 80 \\
Pred. 1 & 65 & 298 \\
\hline
\end{tabular}

\begin{tabular}{lll}
\hline & \multicolumn{2}{l}{ Measured } \\
& 0 & 1 \\
\hline 0 & 2351 & 87 \\
1 & 46 & 92 \\
\hline
\end{tabular}

\begin{tabular}{lll}
\hline & \multicolumn{2}{l}{ Measured } \\
& 0 & 1 \\
\hline 0 & 2376 & 11 \\
1 & 38 & 16 \\
\hline
\end{tabular}

In our tool, when the quality prediction model produces an alarm for roughness, the product will be compared to the corresponding group of good products automatically. Among all process variables, We selected the observed ones based on their importance in the prediction model; high impact in the model correlates with the impact in the defect prediction, and thus, high impact variables are the best candidates to cause the defect, should the distance to the good ones be significant. Parallel coordinates [25] visualize effectively the difference between the selected product and the pool of good products. Figure 4 present the difference between the observed product and the pool of good products (grey) in two cases. True positive product (solid black) diverges from the good products for several variables, while false positive (dashed black) looks quite similar to the good ones.

The parallel coordinate visualization is available for the user as well. Thus, it is possible to learn, what are the factors that increase the risk of defects and how to improve the process in the future. Also, the user can select the predictions of the original model for quality visualization, if preferred.

After the distance calculation, our method produced the alarm only if the product had variable values diverging from the corresponding ones of the good products. As a result, we were able to reduce the number of false positives. Table 3 presents the final results for different product groups. One product group with no defected products or false positives has been left out. There are the results for both validation and test set, because, the number of defected products was quite low for the test set. It can be seen that the number of false positives (FP) can be reduced significantly without losing true positives (TP). The group 4 is especially susceptible to the defect, and there is a definite need for the quality prediction model in manufacturing. In the test set, the AI enhanced prediction model will reduce the percentage of FPs from $48 \%$ to $20 \%$. In validation set the percentage is dramatically better.

TABLE 3. THE RESULTS IN THE NUMBER OF REDUCED FALSE POSITIVES AND THE PRESERVED TRUE POSITIVES FOR VALIDATION (UPPER) AND TEST (LOWER) SETS.

\begin{tabular}{lllll}
\hline & GROUP1 & GROUP2 & GROUP3 & GROUP4 \\
\hline FP & $12 \rightarrow 1$ & $8 \rightarrow 2$ & $9 \rightarrow 4$ & $17 \rightarrow 2$ \\
TP & $5 \rightarrow 5$ & $2 \rightarrow 2$ & $14 \rightarrow 14$ & $71 \rightarrow 45$ \\
\hline FP & $2 \rightarrow 1$ & - & $13 \rightarrow 6$ & $15 \rightarrow 2$ \\
TP & - & - & - & $16 \rightarrow 8$ \\
\hline
\end{tabular}

\section{Conclusions}

In this paper, we present an AI based method for alarm management in situations where the performance of the prediction models is weak because of lacking information. This method is useful especially when predicted alarms are needed quite early in order to be able to adjust variables causing the trouble. The application is from industrial environment, where the utilization of multiple sensors data is common, and thus, it can provide cross-discipline solutions to challenges in smart environments as well. Analogous applications can be found in smart city environment, where monitoring systems can produce alarms when scarce resources should be saved, for example. 


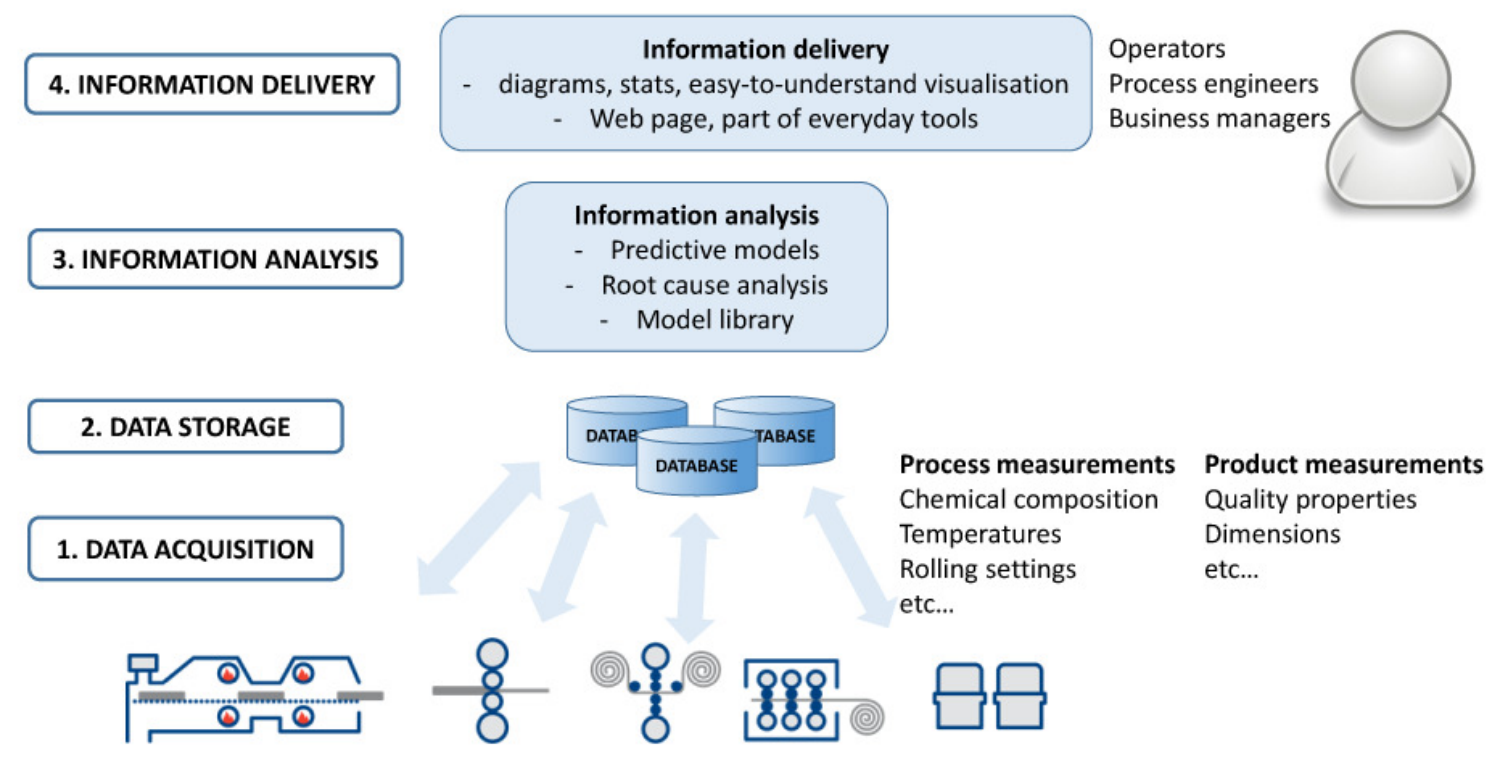

Figure 2. The prototype of QMT.

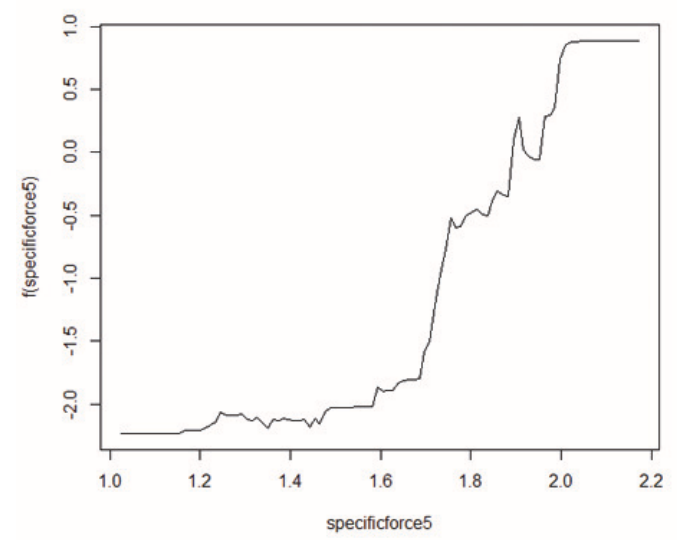

Figure 3. The visualization of the effect of specific force on fifth pass on surface roughness.

Our method enables the automatic improvement of the usability of the defect risk predictions made at the early state of the manufacturing process, when the accuracy of the models suffers because of the lack of information of the future process steps. This way, the products with high risk can be recognized and cost-effectively rerouted. Furthermore, the user can find root-causes for the reduced quality and learn how to improve the process.

The web based quality monitoring tool provides decision support for users in different roles in manufacturing. Currently, the tool is in online test use at Outokumpu, Tornio.
The test period provides valuable users' feedback that can be utilized in the further development of the tool. Users will evaluate also the possibility to move towards fully automatic process control with cognitive systems that are based on smart decision support with statistical prediction models.

\section{Acknowledgments}

The Quality Monitoring Tool was developed in a cooperation with VTT Technical Research Centre of Finland, Ltd.

\section{References}

[1] K. Su, J. Li, and H. Fu, "Smart city and the applications," in Proc. International Conference on Electronics, Communications and Control (ICECC 2011), 2011, pp. 1028-1031.

[2] G. Hancke, B. Silva, and G. Hancke, "The role of advanced sensing in smart cities," Sensors, vol. 13, pp. 393-425, 2013.

[3] H. Chourabi, T. Nam, S. Walker, R. Gil-Garcia, S. Mellouli, K. Nahon, T. Pardo, and H. Scholl, "Understanding smart cities: an integrative framework," in Proc. Hawaii International Conference on System Sciences, 2012, pp. 2289-2297.

[4] L. Filipponi, A. Vitaletti, G. Landi, V. Memeo, G. Laura, and P. Pucci, "Smart city: an event driven architecture for monitoring public spaces with heterogeneous sensors," in Proc. International Conference on Sensor Technologies and Applications, 2010, pp. 281-286.

[5] J. Jin, J. Gubbi, S. Marusic, and M. Palaniswami, "An information framework for creating a smart city through internet of things," IEEE Internet of Things Journal, vol. 1, no. 2, pp. 112-121, 2014.

[6] I. Dumitrache and S. Caramihai, "The intelligent manufacturing paradigm in knowledge society," in Knowledge Management. InTech, 2010, pp. 36-56. 


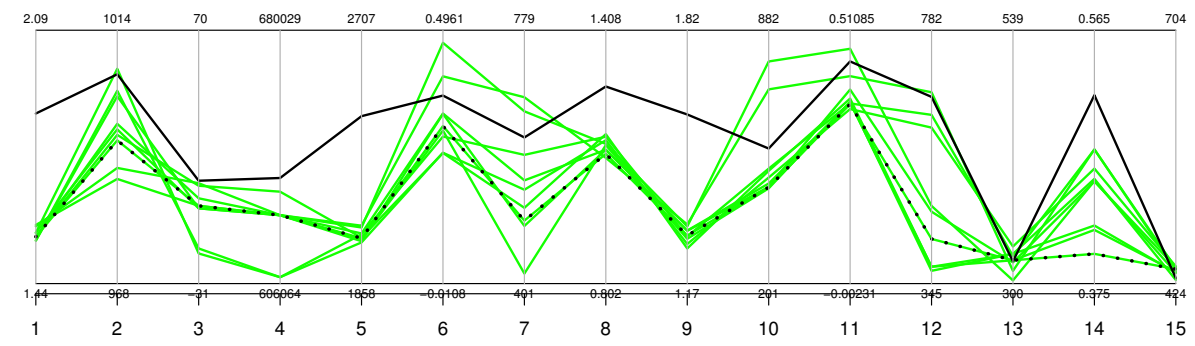

Figure 4. The parallel coordinates visualize the difference between the good products and the observed product having an increased predicted risk for failure (good products (grey), observed true positive (solid black), false positive (dashed black).

[7] I. Juutilainen, S. Tamminen, and J. Röning, "A tutorial to developing statistical models for predictiong disqualification probability," in Computational Methdos for Optimizing Manufacturing Technology, Models and Techniques. USA: IGI Global, 2012, pp. 368-399.

[8] P. Siirtola, S. Tamminen, E. Ferreira, H. Tiensuu, E. Prokkola, and J. Röning, "Automatic recognition of steel plate side edge shape using classification and regression models," in Proc. 9th Eurosim Congress on Modelling and Simulation (EUROSIM 2016), 2016.

[9] E. Gilman, A. Keskinarkaus, S. Tamminen, S. Pirttikangas, J. Röning, and J. Riekki, "Personalised assistance for fuel-efficient driving," Transportation Research Part C: Emerging Technologies ,, vol. 58, pp. 681-705, 2015.

[10] J. Harding, M. Shahbaz, Srinivas, and A. Kusiak, "Data mining in manufacturing: A review," Journal of Manufacturing Science and Engineering, vol. 128, pp. 969-976, 2006.

[11] G. Phillips-Wren, "Intelligent decision support systems," in Multicriteria Decision Aid and Artificial Intelligence. Chichester, UK: John Wiley \& Sons, Ltd, 2013.

[12] O. Logunova, I. Matsko, I. Posohov, and S. Luk'ynov, "Automatic system for intelligent support of continuous cast billet production control processes," International Journal of Advanced Manufacturing Technology, vol. 74, pp. 1407-1418, 2014.

[13] M. Kano and Y. Nakagawa, "Data-based process monitoring, process control, and quality improvement: Recent developments and applications in steel industry," Computers \& Chemical Engineering, vol. 32, no. $1-2$, pp. $12-24,2008$

[14] L. Xu, W. He, and S. Li, "Internet of things in industries: A survey," IEEE Transactions on Industrial Informatics, vol. 10, no. 4, pp. 22332243, 2014.

[15] R. Parasuraman and D. Manzey, "Complacency and bias in human use of automation: An attentional integration," Human Factors, vol. 52, pp. 381-410, 2010.

[16] J. Lee and K. See, "Trust in automation: Designing for appropriate reliance," Human Factors, vol. 46, pp. 50-80, 2004.

[17] H. Haider, "The role of information reduction in skill acquisition," Cognitive Phychology, vol. 30, pp. 304-337, 1996.

[18] H. Bhadesia, "Neural networks in materials science," ISIJ International, vol. 39, no. 10, pp. 966-979, 1999.

[19] S. Tamminen, I. Juutilainen, and J. Röning, "Exceedance probability estimation for quality test consisting of multiple measurements," Expert Systems with Applications, vol. 40, pp. 4577-4584, 2013.

[20] G. Seni and J. Elder, Ensemble Methods in Data Mining: Improving Accuracy Through Combining Predictions, ser. Synthesis Lectures on Data Mining and Knowledge Discovery, R. Grossman, Ed. USA: Morgan \& Claypool, 2010.
[21] A. Natekin and A. Knoll, "Gradient boosting machines, a tutorial," Frontiers in Neurorobotics, vol. 7, 2013.

[22] J. Elith, J. Leathwick, and T. Hastie, "A working guide to boosted regression trees," Journal of Animal Ecology, vol. 77, pp. 802-813, 2008.

[23] J. Friedman, "Stochastic gradient boosting," Computational Statistics and Data Analysis, vol. 19, pp. 367-378, 2002.

[24] S. Tamminen, E. Ferreira, H. Tiensuu, H. Helaakoski, V. Kyllönen, J. Jokisaari, E. Puukko, and J. Röning, "An online quality monitoring tool for information acquisition and sharing in manufacturing: requirements and solutions for the steel industry," International Journal of Industrial and Systems Engineering, 2018, accepted.

[25] A. Inselberg, "Visual data mining with parallel coordinates," Сотриtational Statistics, vol. 13, no. 1, pp. 47-63, 1998. 